\title{
The classification and integration of edges as critical to the perception of reflectance and illumination
}

\author{
ALAN GILCHRIST \\ Institute for Cognitive Studies, Rutgers University, Newark, New Jersey 07102 \\ and \\ STANLEY DELMAN and ALAN JACOBSEN \\ State University of New York, Stony Brook, New York
}

\begin{abstract}
A pattern of luminances equivalent to that of a traditional simultaneous lightness display (two equal gray squares, one on a white background and the other on an adjacent black background) was presented to observers under two conditions, and matches were obtained for both perceived reflectance and perceived illumination level of the squares and their backgrounds. In one condition, the edge dividing the two backgrounds was made to appear as the boundary between a white and a black surface, as in the traditional pattern. The squares then were perceived as almost the same shade of middle gray. In the other condition, a context was supplied that made the edge between the backgrounds appear as the boundary between two illumination levels, causing one square to appear black and the other white. These results were interpreted as a problem for local ratio theories, local edge theories, and lateral inhibition explanations of lightness constancy, but as support for the concepts of edge classification, edge integration, and the retinal image as a dual image.
\end{abstract}

Theories that appear different or even opposite may nonetheless share a common starting assumption, and the preoccupation with such apparent differences may serve to obscure flaws in the common assumption. Believing this to be true for theories of lightness perception, we have chosen an alternative, and somewhat radical, starting point for the experiments we report here. This approach will be more easily grasped if we begin by reviewing the central role of luminance information in current theories of lightness perception.

\section{The Photometer Metaphor: Absolute Luminance Levels as the Basic Input}

Implicit in most theory and research on the perception of surface lightness is a conception we would call the photometer metaphor. This term refers to the assumption that, fundamentally, the visual system measures the intensity of light reflected by each point in a visual scene. Helmholtz and Hering, although their views were usually in opposition, both assumed that absolute intensity of reflected light (luminance) was available as an input to the visual system. Of course, neither their theories of lightness perception nor the more modern theories are

The authors gratefully acknowledge the support of the $\mathrm{Na}$ tional Science Foundation (Grant BNS 77-02655) for this research. A. Gilchrist's mailing address is: Department of Psychology, Rutgers University, Newark, New Jersey 07102. simple photometer theories, since the simple photometer analogy failed right at the outset. The two most widely discussed phenomena of lightness perception, lightness constancy and lightness contrast, show directly that perceived lightness does not correspond to luminance per se. Despite this problem, the photometer metaphor has not been given up. Rather, an attempt has usually been made to rescue it by auxiliary mechanisms. Helmholtz believed that luminance values were placed within an illumination frame of reference by means of an unconscious inference. But the mechanism favored by Hering and most contemporary theorists (Cornsweet, 1970; Jameson \& Hurvich, 1964) has been that of contrast. It is proposed that physiological responses to luminance values are changes through lateral inhibition among cells in the visual system in such a way as to produce a direct relationship between physiological response and perceived lightness.

This approach is best illustrated by considering a classic example of contrast, often used in textbooks. Two pieces of identical gray paper are placed on adjacent white and black backgrounds, respectively. Although the gray pieces have the same luminance (given uniform illumination) the one on the white background appears a slightly darker gray than the one on the black background. A typical contrast explanation goes as follows. Cells receiving light from the two gray pieces are equally ex- 
cited, and would produce equal levels of neural activity if the gray pieces were on the same background. However, cells that receive light from the white background, because of their intense stimulation, are able to inhibit the firing of cells receiving light from the gray piece surrounded by the white. Consequently, the gray piece on the white background appears darker, since the black background does not allow inhibition of those cells receiving light from the gray paper it surrounds.

Notice that this approach takes luminance levels as its starting point. These levels are then altered by contrast mechanisms. The same approach has been extended to explain the constancy of perceived shades of gray seen under varying levels of illumination. In essence, the argument has it that when the general level of illumination is increased, for example, the tendency toward greater excitation of visual cells due to the higher luminances of light entering the eye is canceled by the increased levels of lateral inhibition acting on each cell by its neighbors. The main difference between the two most widely accepted contrast theories would seem to concern whether the increased excitation and the increased inhibition do (Cornsweet, 1971) or do not (Jameson \& Hurvich, 1964) exactly cancel each other out.

The main point is that current explanations of both contrast and constancy are based on the photometer metaphor. If this point is not yet sufficiently clear, perhaps the description of an alternative conception will help to clarify matters.

\section{The Relational Approach: Luminance Gradients as the Basic Input}

According to a more relational, or gestalt, view of lightness perception, the visual system is engaged in a comparison of light rather than a measurement of light. Consider Wallach's (1948) classic experiments on perceived lightness. His elegant disk/ring experiments demonstrated that perceived lightness does not correlate with luminance, but rather correlates with the luminance ratio ${ }^{1}$ between the disk and the ring. What is important is the luminance relationship between the disk and the ring, not the absolute luminance of either. This suggests that, somehow, the two luminances are being compared. Now, it is possible to account for Wallach's results using both a photometer concept and a comparison concept. We might argue that the visual system first measures these two luminances and then compares them. But it is much simpler to imagine that the comparison is performed in a single operation that never involves a luminance measurement.

Recent research involving stabilized retinal images has shown that continual displacement of the optical pattern across the retinal surface is a neces- sary condition for vision. When an unchanging pattern of light continually falls on the same receptor cells, the visual field fades to complete homogeneity within 1 to $3 \mathrm{sec}^{2}$ (Barlow, 1963; Yarbus, 1967). This finding, by itself, appears to challenge the photometer metaphor. A nonuniform pattern of light continues to stimulate the retinal receptor cells, despite the cessation of visual experience. Why is the visual system not able to measure the luminance of the various parts of the image simply because the image no longer moves across the retina? (One might even expect that this stability ought to actually facilitate the measurement of light within the image.)

A potential answer is suggested in a simple experiment performed by Krauskopf (1963). He used a display consisting of a disk of one color, say green, surrounded by a ring of another color, say red, and showed that when the green/red boundary is retinally stabilized (the outer boundary of the red ring is not stabilized and remains visible), it disappears and the entire large disk that is now seen appears homogeneously red.

Consider the fact that even the center of the large disk now appears red, despite the fact that the corresponding receptor cells are still being stimulated with green light. Apparently, the light within a homogeneous region of the image does not provide the basis for the perception of color within that region. This result suggests, quite forcibly, that (at least for nonflickering displays) the visual system picks up the color of a surface from information about the change in light at the surface boundary. Apparently this information can be picked up only when receptor cells are allowed to move across the boundary.

Returning to the earlier Wallach experiment, we can now see that the disk/ring luminance ratio could be picked up directly by the change in stimulation of a receptor cell moving across the disk/ring boundary, without any measurement of the luminance of light in either the disk or the ring. In other words, when receptor cells move across an edge, the effective information may be simply the proportion by which their stimulation has been either reduced or increased. Thus, it is possible that the neural pattern produced when receptor cells move across an edge from a region of one unit of light to a region of five units of light might be exactly the same as that produced if they moved from 10 units to 50 units.

\section{Additional Processes Required by the Relational Approach}

Despite the wide range of evidence (Krauskopf, 1963; Land \& McCann, 1971; Walraven, 1976; Whittle \& Challands, 1969; Yarbus, 1967) support- 
ing the view that the color of a surface is extracted from the change in light at edges, this simple conception will have to be qualified in at least two important ways. One (1) concerns the constancy of surface color even when the surface is viewed against different backgrounds, and the other (2) concerns the fact that not all boundaries in the retinal image represent changes in surface color; some represent changes in illumination.

(1) The change in light at the edge of a surface, by itself, is not a reliable indicator of the color of the surface. The perceived color of a surface can remain approximately constant ${ }^{3}$ despite gross changes (in both direction and magnitude) in the change in light at its boundary. This occurs regularly in everyday life when a surface is successively viewed against backgrounds of different colors. The change in light at the edge of the surface varies, of course, with every change in background color. Conversely, a constant edge ratio can produce a different perceived color or lightness, depending on the color or lightness of its background. For instance, a white paper of $90 \%$ reflectance surrounded by a light gray paper of $30 \%$ reflectance will have the same edge ratio (namely, $3: 1$ ) as a very dark gray paper of $9 \%$ reflectance surrounded by a black paper of 3\% reflectance. Yet, under normal viewing conditions, the white paper and the dark gray paper will never appear even close to the same shade of gray.

Therefore, information about the change in light at the edge of a surface must somehow be integrated with information about the color of the background surface, which is itself probably also derived from the change in light at the edge of the background. The kind of integration of edge information necessary to solve this problem has been proposed by Arend (1973), Gilchrist (1979), and Land and McCann (1971). Such an integration process would allow each field part to be compared with any other field part whether or not the two parts were adjacent to each other in the image. As Land has suggested, the luminance ratio between two spatially remote surfaces can be obtained simply by taking the product of the ratios at each of the edges located between the two surfaces.

(2) Many of the changes in light (both chromatic and achromatic) at boundaries in the retinal image are both caused by, and perceived as, changes in the color and/or intensity of the illumination shining on a surface. Often such illumination edges are gradual, but they are also frequently sharp. If these changes in light were invariably computed by the visual system tu define the surface color of the regions they bound, gross surface color illusions would occur. Constancy of surface color would be impossible, since the visual system would be unable to keep information about the color of surfaces separate from information concerning the color of the illumination. Thus, it seems inescapable, on logical grounds, that if color perception is governed by edge information there must be some process whereby reflectance changes are distinguished from illumination changes.

\section{The Extraction, Classification, Integration Model}

Gilchrist (1979) has proposed a three-process model that would accommodate the crucial role of edge information as well as the two qualifications just discussed.

(1) Extraction. Information about relative luminance and color of adjacent parts of the visual field is extracted as the retina displaces across the retinal image.

(2) Classification. At some point, a decision must be made for each edge in the retinal image, as to whether it is the projection of a reflectance edge in the physical world or an illumination edge.

(3) Integration. Once classified, edges can now be integrated within classes, creating two representations of the scene, one for surface color and one for the pattern of illumination.

\section{The Concept of Two Images in One}

Notice that, according to this model, the retinal image is analyzed into two component images. This idea is sharply at variance with most current theories of lightness perception, but it seems quite consistent with daily experience. It seems self-evident that we not only perceive the gross level of illumination of a scene, but that we also perceive the pattern of illumination within the scene. A curious fact of current lightness theories is the rather unanimous position, either implicit or explicit, that illumination is not perceived. This rejection of illumination perception has not come from perceptual experience; nor has it come from data, which are scanty at best. Rather, it can be traced to the basic photometer metaphor, the concept of the eye as a measuring device. Implicit in the photometer metaphor is the assumption that each point in the visual field can have only one value. Given this artificial constraint, it seems most reasonable to reserve that value for perceived surface color. But perception of both the color and intensity of the illumination, in addition to perception of surface color, suggests that each point has at least two values. Perception of transparency involves the same kind of complexity. These examples have never been adequately handled by theories of color and lightness perception, due, in our opinion, to reliance on the underlying photometer metaphor.

Edge theories, on the other hand, are ideally suited to account for such dualities in perception. 
As the Krauskopf experiment shows, the perceived color of a point is not defined by any dimension of the light itself at that point, but, rather, it is defined by reference to the boundary that surrounds the point. Thus, a point can have more than one value simply by falling within more than one boundary. For example, take the case of a shadow that falls across a floor, partly covering a piece of white paper. The luminance of a given point located on the paper and within the shadow is, of course, a product of both the illumination level within the shadow and the reflectance of the white paper. But that luminance is not what we perceive. Rather, we perceive two things: the lightness of the paper, which is determined by the change in light at the edge of the paper (in conjunction with other reflectance edges in the larger context), and the brightness or dimness of the shadow, which is determined by the change in light at the boundary of the shadow (again in conjunction with other illumination edges). Examples like this one are not exceptions; they are the rule in natural scenes. In fact, Gilchrist (1979) has proposed that it is useful to think of the retinal image as analyzed into two subimages, one representing the pattern of illumination and one representing the pattern of surface color. Very similar concepts have been described by Barrow and Tenenbaum (1978) and Bergstrom (1977).

The extraction/classification/integration model is undoubtedly too sterile and machine-like to be taken very literally. Its value lies, rather, in the identification of processes that would appear, on logical grounds at least, as necessarily required of an adequate theory of surface-color perception. Nevertheless, this model has been subjected to empirical test in the present research, especially the two qualifications of the edge concept discussed above. That is, we have asked if it can be shown that spatially separate edge signals are integrated by the visual system, and if it can be shown that the processing of reflectance edges is different from that of illumination edges. (The question of what cues provide a basis for the classification process is beyond the scope of this paper. Here we hope only to help establish the importance of this question.)

This kind of test requires that the perceptual organization of a display be changed while contrast explanations are excluded by holding both the retinal pattern and the luminances of the display constant. A number of researchers (Beck, 1965; Gogel \& Mershon, 1969; Hochberg \& Beck, 1954; Kardos, 1934; Mershon, 1972) have produced lightness changes while satisfying these conditions by varying the perceived three-dimensional spatial position of part of the display. See Gilchrist (1980) for a review of these studies. MacLeod (1932) produced analogous results without changing perceived spatial position by showing that a target disk appears lighter when lying on a shadowed light gray surface than when lying on a nonshadowed dark gray surface of the same luminance. This result shows that the lightness of the target disk is not determined simply by the luminance ratio at the boundary of the target disk, but, rather, that this luminance ratio becomes meaningful only in relation to the boundary of the background. Unfortunately, MacLeod allowed the sharpness of the background boundary to change by using a penumbra to signal the presence of the shadow. As MacLeod's (1947) own later work showed, changes in the sharpness of a gradient can influence perceived lightness without necessarily producing a qualitative change in perceptual organization.

The logic of our experiment involves comparing the appearance of one display composed entirely of different reflectances with the appearance of a second display, identical in geometry and photometry to the first display, but perceptually composed of both different reflectances and different levels of illumination. Like MacLeod, we did not use changes in spatial position to create the perceptual reorganization, but, unlike MacLeod, we prevented a change in edge sharpness.

We selected as our basic display the traditional lightness-contrast pattern consisting of two squares of equal gray centered on white and black backgrounds, respectively. Our task was to create a second, identical display in which the boundary between the white and black background regions appeared as the boundary between two levels of illumination falling on the display. In edge terminology, we were substituting an illumination edge for a reflectance edge. Hence, we refer to this kind of experiment as an edge-substitution experiment.

In addition, our experiment offers a direct comparison of the two central phenomena in lightness perception: contrast and constancy. Inherent in the experimental design is a test of the possibility that the same contrast mechanism currently used to explain simultaneous lightness contrast can be also used to explain lightness constancy.

\section{EXPERIMENT 1}

\section{Method}

A rectangular piece of middle gray (reflectance $=16 \%$ ) paper, 9 in. $(22.9 \mathrm{~cm})$ high $\times 12$ in. $(30.5 \mathrm{~cm})$ wide was suspended 44 in. $(112 \mathrm{~cm})$ above the floor and 24 in. $(61 \mathrm{~cm})$ in front of a wall. A small black square (reflectance $=3.1 \%), 2$ in. $(5.1 \mathrm{~cm}) \times$ 2 in., was placed on the gray rectangle, slightly to the left of center, and a white square (reflectance $=90 \%$ ) of the same size was placed to the right of the center. These two squares will be referred to as the targets, and the gray rectangle with the two squares on it will be referred to as the display. The main 


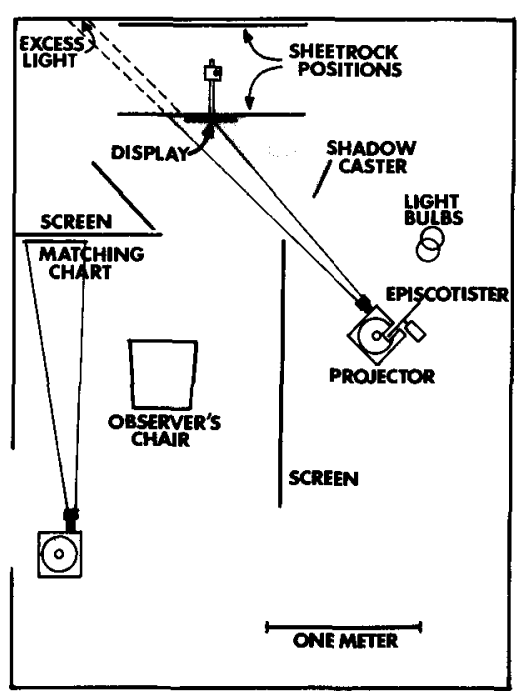

Figure 1. Floor plan showing experimental arrangements.
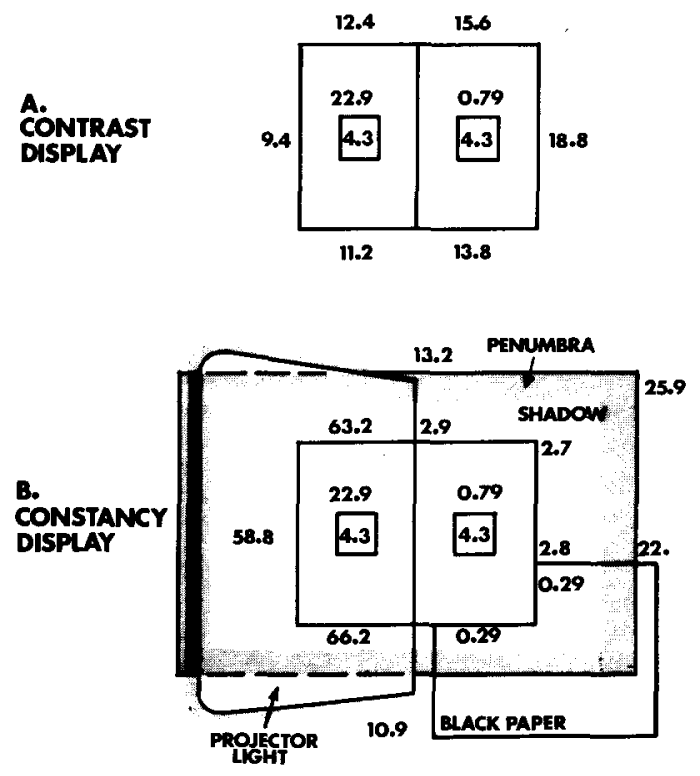

Figure 2. Diagram of the displays used in Experiment 1. The numbers represent luminances in footlamberts.

source of light in the room was provided by two incandescent $300-$ W light bulbs, located 33 in. ( $84 \mathrm{~cm}$ ) in front of and 55 in. $(140 \mathrm{~cm})$ to the right of the center of the display. A piece of black paper was suspended in midair, 32 in. $(81 \mathrm{~cm})$ from the light bulbs, so as to cast a shadow over the entire display. The purpose of the shadow was to make the right half of the gray rectangle appear black (as in the Kardos illusion) in the completed display. The left half of the display was strongly illuminated with a trapezoidal projection of light from a Carousel slide projector located 55 in. $(140 \mathrm{~cm})$ in front of and 35 in. $(89 \mathrm{~cm})$ to the right of the center of the display. The purpose of this light was to make the left half of the gray rectangle appear white (as in the Gelb effect) in the completed display.

Since the light from the two incandescent bulbs was more orange in color than the light from the projector, a rotating Plexiglas disk containing eight 7-deg segments of orange ace- tate (and eight 24-deg opaque segments for intensity adjustment) was inserted between the quartz-halogen projector bulb and the condensor lens. The Plexiglas disk (or episcotister) and its motor were mounted on a table separate from that of the projector to prevent vibration of the slide projector and allow a sharp focus on the right-hand boundary of the projected light.

The observer was seated $65 \mathrm{in} .(165 \mathrm{~cm})$ in front of and slightly to the left of the display. The projector setup, the two light bulbs, and the shadow caster were hidden from the observer by a black plastic screen, 67 in. $(170 \mathrm{~cm})$ wide $\times 96$ in. $(244 \mathrm{~cm})$ high, located $31 \mathrm{in}$. $(79 \mathrm{~cm})$ to the right of the observer. Those parts of the trapezoidal projection of light, and the cast shadow, that overshot the gray rectangle were also hidden from the observer by a screen 33 in. $(84 \mathrm{~cm})$ wide and 78 in. $(198 \mathrm{~cm})$ high, located 30 in. $(76 \mathrm{~cm})$ in front of and parallel to the gray rectangle itself. This screen was covered with black velvet.

A two-dimensional matching chart, 17 in. $(44 \mathrm{~cm})$ wide $x$ 18 in. $(46 \mathrm{~cm})$ high, was attached to the front of this screen at approximately the same height as the display. The chart contained nine rows of Munsell chips on a white background. Each row contained 16 chips, from black (on the left) to white. Each chip was $1 / 2$ in. square and had a 1/8-in. black border on the top and left side. A slide containing step functions of nine graded neutral densities was projected onto the chart so that each row of Munsell chips received a different level of illumination, with bright illumination on the top row and dim illumination on the bottom. This chart allowed the observers to select a chip that matched a part of the display in both reflectance and illumination. Pieces of black velvet were suspended near the matching chart in a sort of tent-like arrangement in order to prevent stray light from illuminating the chart.

Behind the screen supporting the matching chart, a rigid reflecting panel, 24 in. $(61 \mathrm{~cm})$ wide $\times 48 \mathrm{in}$. $(122 \mathrm{~cm}) \mathrm{high}$, was mounted at a 45-deg angle to the display. This panel could be covered with varying amounts of white, black, or colored paper in order to finely tune the illumination falling on the shadowed part of the display.

Directly behind the display, a large off-white (reflectance = $60 \%$ ) plasterboard panel, 48 in. $(122 \mathrm{~cm}) \times 48$ in., was placed against the rear wall. It served as the background for the display. The walls and ceiling of the room were all painted matte black to control reflected light.

These arrangements produced the appearance of a traditional simultaneous lightness contrast pattern (gray squares on white and black backgrounds) standing in the same illumination as that of the surrounding vicinity of the room. This display was used in what we refer to as the contrast condition of the experiment. The other condition, which we call the constancy condition, was created by making one change. A rectangular piece of black paper was attached to the Sheetrock panel (see Figure 2), and the panel was moved forward to a position immediately behind, and in fact touching, the gray rectangle. This panel served to reveal the special illumination conditions, since the outlines of both the projected light and the cast shadow were visible on the surface of the panel. In terms of the logic of the experiment, the panel served to cause the edge that divided the respective backgrounds of the two square targets to appear as a change in illumination rather than as the boundary between white and black paper. But it is important to note that the presence of the plasterboard background was not allowed to change the pattern of luminances within the gray rectangle. The black rectangular piece of paper served to make the conditions of illumination more visually clear.

Observers. A total of 18 naive undergraduate students served voluntarily as observers, 9 in the contrast condition and 9 in the constancy condition.

Procedure. The observer was first seated and, after the two dimensions of the matching chart had been explained, asked to give a simple verbal description of the display. Then the matches were made. The observer was first asked to select a matching illumination level (or levels), and then reflectance 
matches were made from the row of chips located within the matching illumination level. After the matches had been recorded, the actual experimental arrangements were- revealed to the observer and he or she was further questioned in order to confirm and clarify the experimenter's understanding of the original verbal description.

\section{Results}

The median reflectance and illumination matches are shown in Figure 3. The results of the contrast condition make it clear that our display, created in part with different levels of illumination, did successfully replicate the appearance of the standard simultaneous lightness pattern. The left and right backgrounds appeared fully white (median, 9.5; mean, 9.4; SD, 0.17) and black (median, 2; mean, 2.6; SD, 1.49), respectively. The targets appeared middle gray, with the right-hand target (median, 5; mean, 5.6; SD, 1.34) appearing about one-half a Munsell step lighter than the left-hand target (median, 4.5; mean, 4.7; SD, 1.00), a contrast effect that is consistent with results we have repeatedly obtained using the standard pattern constructed solely out of paper, although the difference between means for the two targets falls just short of significance $[t(8)=1.61, p<.01]$. At the same time, eight of the nine observers gave a lighter rating to the target on the right, while the ninth observer rated the targets as equal.

The display was perceived as uniformly illuminated by all nine observers, at a level close to that of the actual room illumination at the position of the display (ignoring the shadow and the projected light), For instance, the luminance of the left-hand background was $22.9 \mathrm{fL}$, and the luminance of the corresponding median matching chip (9.5) under the median matching illumination (row $C$ ) was $18.5 \mathrm{fL}$.

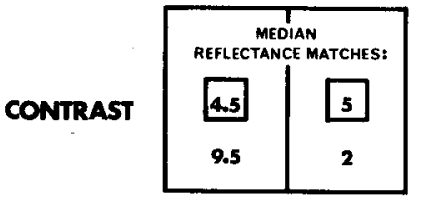

MEDIAN ILLUMINATION MATCHES:

C

B
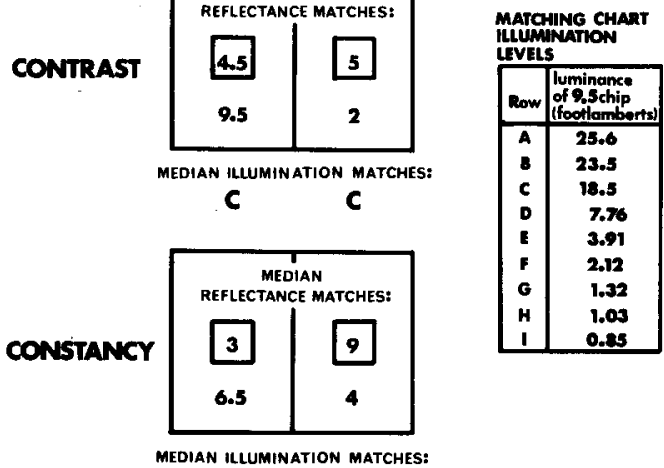

Figure 3. Median reflectance and illumination matches. Reflectance matches are given in Munsell values. Black is 2 and white is 9.5 on the Munsell scale. Chart at right shows luminances in footlamberts of white chip under each of the nine levels of illumination in the matching chart.
In the constancy condition, the perceived organization of the display was markedly different. This is seen very clearly in the reflectance matches made to the two targets. The left-hand target appeared black (median, 3; mean, 3.06; SD, 0.73) and the right-hand target appeared white (median, 9; mean, 8.6; SD, 0.93), a difference that is significant far beyond the .001 level $[t(8)=13.9]$. Most, but not all, of the perceived difference in background reflectance disappeared (left backgroundmedian, 6.5; mean, 6.6; SD, 0.73; right background-median, 4 ; mean, 4.3 ; SD, 0.73 ). The remaining difference is significant $[\mathrm{t}(8)=6.56, \mathrm{p}<$ $.001]$. In its place a large, significant difference $[t(8)=7.94, p<.001]$ in the illumination levels of the two backgrounds was perceived (left backgroundmedian, 23.53; mean, 20.8; SD, 5.53; right background-median, 1.32; mean, 1.75; SD, 2.05). In fact, the ratio between the illumination matches made to the left and right sides, 17.8 to 1 , was about $61 \%$ of the actual left-to-right illumination level ratio in the display. The matching illumination levels were somewhat darker $(0.73 \mathrm{log}$ units lower for the left side and $0.51 \mathrm{log}$ units lower for the right) than the actual illumination levels in the display, owing possibly to the dark velvet background against which the matching chart was viewed. For both targets and both backgrounds, the constancy condition produced mean reflectance matches that were significantly different $(p<.005)$ from those of the contrast condition.

\section{Discussion}

Consider first what ideas are not supported by these results.

(1) Luminance as the correlate of lightness. It comes as no surprise that these results demonstrate again the fact that the perceived lightness of a surface does not depend on its luminance level. The four target squares in the two conditions appeared as various shades of gray, ranging from approximately black (Munsell 3) to white (Munsell 9) even though each target square always had the same luminance of $4.3 \mathrm{fL}$.

(2) Luminance ratios as the correlate of lightness. A comparison of the results from the contrast and constancy conditions demonstrates that perceived lightness does not depend upon ratios of luminance, if what is meant by ratios is simply the local ratio between a target and its background. For example, the left-hand target appeared middle gray (Munsell 4.5 ) in the contrast condition but black (Munsell 3) in the constancy condition, yet the targetto-background ratio was the same in both cases. An even greater discrepancy (5 vs. 9) was found for the right-hand target.

(3) Edge change as the correlate of lightness. The same logic used for ratios applies equally to the 
possibility that perceived lightness depends simply on the proportion of change in luminance at the edge of a surface. A local ratio theory and a local edge theory are functionally indistinguishable under many conditions. However, they could, in principle, be distinguished using a Craik/O'Brien contour (Cornsweet, 1970, p. 270).

(4) Lightness constancy as reducible to lightness contrast. An issue of long-standing controversy concerns the claim, made early by Hering (1874/ 1964 , p. 140) and endorsed by others (see Freeman, 1967, for a review), that the same inhibitory mechanism that makes a gray paper look darker when it is on a white background also prevents surfaces from appearing lighter gray when the illumination is increased. Given that this claim is so central to the debate between so-called sensory and cognitive theories, it is surprising that no study has incorporated both the contrast paradigm and the constancy paradigm into the same experiment for direct comparison. Our experiment provides such a comparison. If lightness constancy were produced by lateral inhibition, then the amount of inhibition necessary to make a black square in bright illumination look black (left-hand target, constancy condition) would also have to make a gray square on a white background (left-hand target, contrast condition) look black. It should now be quite clear that contrast and constancy effects are of such a different magnitude that the reduction of one to the other is unreasonable.

The fact that the target lightness appeared so different in the two conditions of our experiment, despite identical target and surround luminances, presents a serious challenge to the currently fashionable view that lateral inhibition of visual cells provides an explanation for lightness constancy. As far as the target squares are concerned, the amount of inhibition (as well as excitation) present in that part of the visual field must essentially be the same in both conditions of our experiment; yet the perceptions are very different.

The only possibility for avoiding this conclusion would appear to lie in the fact that the illumination pattern projected onto the plasterboard background in the constancy condition altered the luminances in that part of the field surrounding the gray rectangle. However, it is not at all clear what predictions a contrast theory would make in such a case involving a luminance change in the surround of a surround. For instance, the luminance of the region to the left of the left half of the gray rectangle was raised from 9.4 to $58.8 \mathrm{fL}$ in the constancy condition. This change in luminance could be expected to either darken or lighten the perceived lightness of the left-hand target, depending on what parts of the contrast literature are accepted. For instance, the idea of remote inhibition would require a darkening of the target, since light is, in fact, being added to that target's larger context. On the other hand, the concept of disinhibition (Cornsweet, 1970 , p. 307) would lead to an expected lightening of the target, since increased inhibition acting on cells corresponding to the left half of the gray rectangle would reduce their ability to inhibit cells corresponding to the left-hand targets. This ambiguity in contrast theories, when dealing with anything more complex than a single target/surround pattern, subverts one of the main advantages of a contrast theory, namely, its much-touted clarity and specificity.

Practically speaking, however, we need not worry about the disinhibition argument, because the target, in fact, appeared darker, not lighter, in the constancy condition. As for the argument of remote inhibition, we would not expect much effect, because the plasterboard background is never closer than $1.8 \mathrm{deg}$ of visual angle to the target and contrast effects have been found to drop off precipitously beyond distances of about 1 deg (Leibowitz, Mote, \& Thurlow, 1953). Nevertheless, we created a control condition to lay the argument completely to rest.

\section{EXPERIMENT 2}

\section{Method}

The control condition was the same as the constancy condition of Experiment 1, but with two changes. First, the trapezoidal projection of light was moved to the right until its righthand edge was only $1 / 2$ in. from the right-hand target (see Figure 4). Second, the rectangular piece of black paper that had been behind the lower right-hand corner of the gray rectangle was replaced with a large sheet of black paper that was inserted behind the left half of the display. These changes resulted in a general shift of luminance from the left to the right side of the area surrounding the display. For instance, the luminance of the area surrounding the left half of the gray rectangle was now lower in this control condition than it was in the contrast condition (2.6 fL as opposed to $9.4 \mathrm{fL}$ ). Moreover, the two remaining areas of highest luminance $(66.3$ and $66.4 \mathrm{fL}$ ) where the projector light shone on the plasterboard panel, were now actually closer to the right-hand target than to the left.

If our original constancy results were due to remote contrast

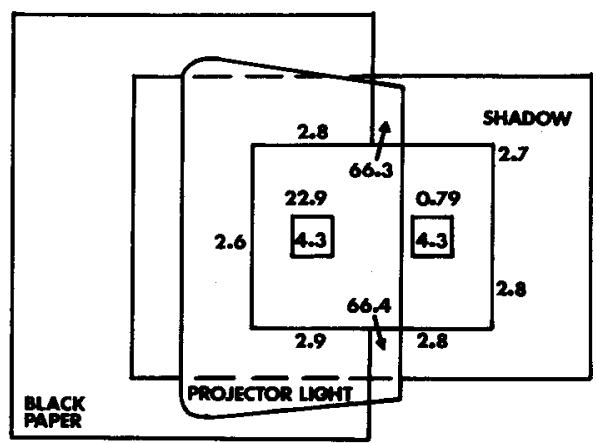

Figure 4. Diagram of display used in Experiment 2, with lnminances shown in footlamberts. 
effects caused by luminance changes on the plasterboard background (rather than to the perceptual reorganizing effect of the context), this control condition would be expected to yield the kind of data we had gotten in the contrast condition of Experiment 1.

The procedure was the same as before for the nine additional observers who made matches under this control condition.

\section{Results}

The results produced by the control condition were, in fact, similar to those obtained in the constancy condition of Experiment 1, not those in the contrast condition. The median Munsell match for the left-hand target was 3 (mean, 3.3; SD, 0.94), exactly as in the original constancy condition, and the match made to the right-hand target was 8.5 (mean, 8.4; SD, 0.65), only half a Munsell step darker than before. These values show no significant differences between this control condition and the previous constancy condition $[\mathrm{t}(8)=0.56$ for both left and right targets]. The reflectance matches made to the backgrounds showed more change (significant for both the left and right backgrounds), with the left-hand background now receiving a median match of 7.5 (mean, 7.8; SD, 0.75), as opposed to 6.5 in the original constancy condition $[t(8)=3.53, p<.01]$ and the right-hand background receiving a 3.5 (mean, 3.5; SD, 0.75), as opposed to a $4[\mathrm{t}(8)=2.30, \mathrm{p}<.05]$. It is interesting to note that this increase in the perceived reflectance difference between backgrounds was accompanied by a perceived decrease in the perceived illumination difference between them. In this condition, the median illumination matches were row $\mathrm{C}$ for the left side (see Figure 3 for conversion to luminances) and row $\mathrm{G}$ for the right side.

\section{GENERAL DISCUSSION}

The fact that the results of Experiment 2 are so similar to those of the constancy condition in Experiment 1 demonstrates that remote inhibition is not the explanation for the results of Experiment 1. There thus appears no way to explain the results of the three conditions of Experiments 1 and 2 using conventional excitation/inhibition concepts, apart from ad hoc arguments.

\section{Cognitive Theories}

Evaluating these results in relation to cognitive theories is more difficult due to the traditional lack of operational definition in cognitive theories. These results might be taken as supporting a Helmholtzian unconscious-inference account, but only in a very general way. Advocates of that school have never been successful in explaining how illumination could be perceived prior to the perception of surface color. In the present display, for instance, the kind of information that produces the perception of surface lightness, namely the direction, magnitude, and organization of edge ratios, also seems to produce the perception of the illumination pattern. It would make as much sense to say that perception of illumination depends on taking surface lightness into account as to use the classic formula, which is the reverse of this.

Rock (1977) has made an important contribution to this line of thought by arguing that, rather than having to make an unconscious inference about the actual amount of illumination of a given surface, it may only be necessary that the visual system be able to infer that the level of illumination on two surfaces (in the same plane, for instance) is the same. This makes the basis of the inference potentially much more specifiable; yet this formulation would fail in the present case, since the key surfaces all lie within the same plane, although they are differentially illuminated.

Beck (1972) has attempted to merge sensory and cognitive approaches. He has proposed that, after neural signals are altered by lateral inhibitory interactions, they are assimilated to a cognitive schema. The present results could presumably be considered as consistent with Beck's theory. The limitation of this approach, however, as with earlier cognitive approaches, lies in its lack of specificity. Beck has not thus far clarified what kinds of experimental results would not be consistent with his theory.

\section{Relational Theory}

We turn now to a discussion of the relational approach, and begin by evaluating the degree to which our results provide support for three ideas described in the introduction: (1) the integration of edge ratios, (2) the classification of edge ratios, and (3) the concept of the retinal image as a dual image.

(1) Integration of edges. According to the concept of integration of edges, as we have used it, the ratio of luminances at the edge of a surface does not, by itself, determine the lightness of the surface. That ratio, in our view, contributes only relative information about the relationship between the lightness (assuming a perceived reflectance edge) of the target and surround, not about the absolute lightness of either. Absolute lightness requires that the target/surround relationship be integrated with other relationships, information about which is presumably contributed by other edges in the visual field.

The integration concept has been illustrated qualitatively in a simple experiment by Arend, Beuhler, and Lockhead (1971). Two targets of equal luminance were placed on a single homogeneous background of lower luminance. A Craik/O'Brien contour was then introduced into the background, halfway between the two targets. The illusion-producing 
contour made the targets appear to stand on backgrounds of different lightness. Three perceptual outcomes were possible. (1) The target on the lighterappearing background could appear darker by contrast with its phenomenally lighter background. Or (2) the targets could continue to appear equal, since their edge ratios continued to be equal. In fact, however, (3) the target on the lighter appearing background appeared lighter than the other target. In other words, the luminance ratio between that target and its lighter appearing background retained its integrity; when its background appeared to become lighter, so did the target.

Our results show the same effect. The actual luminance ratio between the left-hand target and its surround was 1:5.3. When that surround appeared white (reflectance $=90 \%$ ) in the contrast condition, the target appeared gray (reflectance $=15.6 \%$ ), yielding a "perceived target/surround ratio" of 1:5.8. But when the same surround appeared light gray (reflectance $=36.2 \%$ ) in the constancy condition, the target appeared black (reflectance = $6.55 \%$ ), yielding a "perceived target/surround ratio" of 1:5.5. This same pattern of results is present for the right-hand square as well. There the actual target/surround luminance ratio was $5.4: 1$, while the "perceived target/surround ratios" were 6.3:1 for the contrast condition and $6.6: 1$ for the constancy condition.

This concept of integration indicates that the perceived lightness of a surface cannot be determined simply by information derived from the edge of the surface itself. The edge dividing the two backgrounds is as critical to the appearance of the two targets as are their own edges. This idea represents commonsense logic and can be illustrated with a simple example. Let us say that Gelb has six times as many marbles as Hering, and Helmholtz has five times as many marbles as Katz. Who has more marbles, Hering or Helmholtz? Obviously the question cannot be answered with just these two pieces of information. One more piece of information is needed, namely the relationship between the marbles of Gelb and Katz. The nature of this relationship will prove to be decisive. If Gelb has 30 times as many marbles as Katz, then Hering and Helmholtz have the same number of marbles. But if Gelb and Katz have the same number of marbles, then Helmholtz has many more marbles than (30 times as many as) Hering.

More direct evidence for edge integration comes from a stabilized-image experiment by Yarbus (1967, p. 97). Red disks of medium lightness were placed on adjacent white and black backgrounds, respectively. This, of course, would make the two disks appear slightly different in lightness. ${ }^{4}$ However, when the difference between these two backgrounds was made to disappear by retinally stabilizing their boundaries, the unstabilized red disks changed radically in lightness, one (on the objectively white ground) turning very dark and the other turning very light. If this experiment were repeated with gray targets rather than red, the integration concept, in its simplest form, would predict that the targets would appear as black and as white, after stabilization, as the backgrounds appeared before stabilization.

The logic of the Yarbus experiment is the same as the logic of the edge-substitution experiment reported here, but the difference is very interesting. In both cases, it can be said that the perceived reflectance relationship between the two target squares was changed by removing the information contributed by the middle, background/background edge from the perceived reflectance representation. In the stabilized-image experiment, however, the information was removed at the point of extraction (and the resulting display appeared uniformly illuminated), while in the edge-substitution experiment, the information was removed at the point of classification (and it showed up in the observer's perception of the illumination). This indicates that, as far as perceived reflectance is concerned, an edge that is classified as an illumination edge is almost as irrelevant as a stabilized edge.

(2) Classification of edges. More often than not, the importance of edges has not been stressed by analyses of lightness, but even when it has been, there has usually been no distinction made between reflectance edges and illumination edges, as if an edge were simply an edge, period. If the distinction between these two classes of edges were not critically important to the visual system, we should have expected no sizable differences in the matches made under the two main conditions. But, in fact, the reflectance matches were dramatically different in the two conditions, and the fact that an 18to-1 perceived illumination difference showed up in the constancy condition, whereas none had existed in the contrast condition, shows that the luminance-change information that was no longer part of the perceived reflectance pattern was not simply ignored, or filtered out, but was, in fact, used in a different way. This reciprocal relationship between the perception of reflectance and illumination edges strongly indicates that any successful theory of lightness perception will have to include reference to the perception of illumination.

There is reason to believe that the pattern of our data would have been even more pronounced had we succeeded in causing the background/background edge to appear completely and exclusively as an illumination edge in the constancy condition. According to the matching data, the luminance ratio at that edge was attributed mostly to a change in illumination, but partly to a change in reflectance. 
It is possible that this result was produced, in part, by the somewhat artificial conditions of the laboratory display. Factors such as the sharpness of the edge and its regular relationship to the other reflectance edges may have encouraged the perception of some reflectance change at that edge.

There is reason to question, however, the degree to which the matching data represents the naive perceptual experience of the display. Eight of our nine observers, in the verbal questioning, reported that they perceived a single gray rectangle of uniform color in spite of the fact that the lightness matches made to the two sides were not equal. This paradox is not unusual in experiments of this kind. It may be that the matching task alters the perceptual attitude of the observer. Rather than taking in the whole display in a natural fashion, the observer may be forced, by the task, to focus in on only a limited part of the display. Given the importance of visual context, it is not hard to imagine that such a reduction of visual scope could change the appearance of a surface.

On the other hand, edges that contain both a component of reflectance change and a component of illumination change do exist in many scenes and they are perceived accordingly. The classification concept need not be interpreted in an exclusive, either/or fashion. What is central to the idea, however, is the notion of a tradeoff. In our experiment, this would mean, ideally, that to whatever extent the background/background edge was not perceived as a change in reflectance, to that same extent it would be perceived as a change in illumination. The actual results are not far from this pattern; $77 \%$, amounting to a 10:1 ratio, of the reflectance change that was perceived in the contrast condition was not perceived in the constancy condition. In its place, an 18:1 ratio of illumination was perceived.

The retinex model. Fundamentally, the work reported here is quite consistent with that of Land (1977). Land has broken with the photometer metaphor in favor of a relational approach. This can be clearly seen in his definition of the correlate of perceived surface color, namely a triplet (or relationship) of three values, each of which represents the relative degree to which a given retinex is stimulated by light from the surface. The integration process that we have discussed is essentially the same as that proposed by Land and McCann (1971). And finally, not only have Land and McCann pointed out the importance of edges, but they also have been careful to make the distinction between illumination and reflectance edges. Our only criticism lies in the fact that they have minimized the classification problem by assuming that illumination edges, because they are often so gradual, are filtered out of the system so that they play no role in lightness perception.
Our constancy display could be considered a simplified version of one of Land's Mondrian patterns. But, instead of casting a gradual illumination edge across the pattern of reflectances, as Land and McCann did, we projected an illumination gradient that was as sharp as the reflectance edges. The high degree of constancy that we obtained suggests that Land and McCann would have gotten the same results as they did even if their own illumination gradient had been sharp. This leaves their theory incomplete, since, by their current model, when the visual system encountered such a sharp illumination edge, it would treat it as a reflectance edge and this would produce sizable lightness illusions. It seems that the classification process we propose would be a good complement to the retinex model.

The basis of classification. The discussion so far has also left our own account of lightness perception incomplete in an important way. Almost nothing has been said concerning the basis on which the classification of edges is made. In part, this is because the complete answer to this question is not yet known, to us at least. However, we believe that we have identified a number of important factors, and these will be presented in a subsequent publication. For present purposes, it can be safely said that the classification is essentially determined by the relationship of the edge to its context.

(3) The retinal image as a dual image. Our use of the two-dimensional matching chart allowed us to measure both the perceived reflectance and the perceived illumination of surfaces at the same time. Although this method undoubtedly cannot be regarded as a direct measure of perception, we hoped that it would provide at least a rough indication of the reflectance and illumination patterns as perceived by our observers so that we could see how the combination of these two empirically derived patterns would compare with the actual luminance distribution of our display.

This comparison is shown in Figure 5. The perceived profiles were created in the following way. The median Munsell matches for the targets and backgrounds were converted into percentages of reflectance, which were, in turn, converted to logarithmic values. These values were then plotted by spatial position to yield what might be called a perceived reflectance profile (upper left and lower right graphs in Figure 5). To create a perceived illumination profile for the constancy condition, luminance measurements taken from the Munsell 9.5 chip within the two median matching illumination levels were converted to logarithmic values and plotted against spatial position (middle left-hand graph). No perceived illumination profile was necessary for the contrast condition, since the illumination was perceived as homogeneous. The actual 
PERCEPTION:

(Contrast Condition)
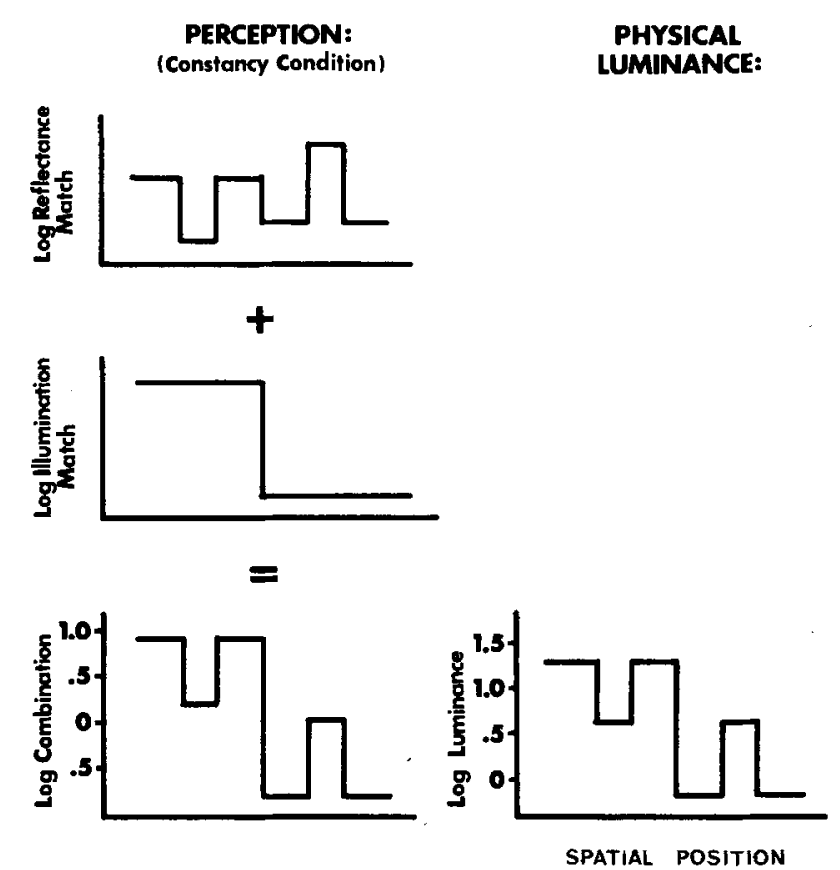

PHYSICAL

LUMINANCE:
Figure 5. A comparison of the actual luminance profile of the display with profiles derived from the matching data. Ordinate values on the bottom row of graphs are luminances in footlamberts. numerical values have not been shown on the ordinates of either the perceived reflectance profile or the perceived illumination profile, since it is the relative values that are important. The purpose of plotting the perceived reflectance and illumination values in this way was to produce a scale that was common to both variables so that the two profiles could be combined, as shown in the lower left-hand graph of Figure 5.

The most interesting feature of Figure 5 is that, in the constancy condition, the pattern of reflectance matches, when combined with the pattern of illumination matches, provides a pattern that is remarkably similar to the actual luminance profile of the display. In the contrast condition, in which the illumination was perceived as uniform, the reflectance matches by themselves closely duplicate the luminance profile. The general conclusion suggested by these results is that the visual system works to account fully for all gradients of light present on the retina, whether by the perception of changes in reflectance, changes in illumination, or both.

\section{REFERENCES}

Arend, L. E. Spatial differential and integral operations in human vision: Implications of stabilized retinal image fading. Psychologicr! Review, 1973, 80, 374-395.

arend, L. E., Buehler, J. N., \& Lockhead, G. R. Difference information in brightness perception. Perception \& Psychophysics, 1971, 9, 367-370.

BARLOW, H. B. Slippage of contact lenses and other artefacts in relation to fading and regeneration of supposedly stable retinal images. Quarterly Journal of Experimental Psychology, 1963, 15, 36-51.

Barkow, H. G., \& Tenenbaum, J. M. Recovering intrinsic scene characteristics from images. In A. Hansen \& E. Riseman (Eds.), Computer vision systems. New York: Academic Press, 1978.

BEck, J. Surface color perception. Ithaca: Cornell University Press, 1972.

BECK, J. Apparent spatial position and the perception of lightness. Journal of Experimental Psychology, 1965, 69, 170-179.

BEnGSTröm, S. S. Common and relative components of reflected light as information about the illumination, color, and three-dimensional form of objects. Scandinavian Journal of Psychology, 1977, 18, 180-186.

Connsweet, T. Visual perception. New York: Academic Press, 1970.

Flock, H. R., \& Noguchi, K. An experimental test of Jameson and Hurvich's theory of brightness contrast. Perception \& Psychophysics, 1970, 8, 129-136.

Freeman, R. B. Contrast interpretations of brightness constancy. Psychological Bulletin, 1967, 67, 165-187.

Gilchrist, A. L. Perceived lightness depends on perceived spatial arrangement. Science, 1977, 195, 185-187.

Gilchrist, A. L. The perception of surface blacks and whites. Scientific American, 1979, 240, 112-124.

Gilchaist, A. L. When does perceived lightness depend on perceived spatial arrangement? Perception \& Psychophysics, 1980, 28, 527-538.

Gogel, W. C., \& Mershon, D. H. Depth adjacency in simultaneous contrast. Perception \& Psychophysics, 1969, 5, 13-17.

Hering, E. Outlines of a theory of the light sense (L. M. Hurvich \& D. Jameson, trans.). Cambridge, Mass: Harvard University Press, 1964. (Originally published 1874.)

HochвEro, J. E., \& BEcx, J. Apparent spatial arrangement and perceived brightness. Journal of Experimental Psychology, 1954, 47, 263-266.

Jameson, D., \& HuRvich, L. M. Theory of brightness and color contrast in human vision. Vision Research, 1964, 4, 135-154. 
KAndos, L. Ding und Schatten. Zeitschrift für Psychologie, 1934, No. 23.

KrAUSKOPF, J. Effect of retinal image stabilization on the appearance of heterochromatic targets. Journal of the Optical Society of America, 1963, 53, 741-744.

LAND, E. H. Retinex theory of color vision. Scientific American, 1977, 237, 108-128.

LAND, E. H., \& MCCANn, J. J. Lightness and retinex theory. Journal of the Optical Society of America, 1971, 61, 1-11.

Leibowitz, H., Mote, F. A., \& Thunlow, W. R. Simultaneous contrast as a function of separation between test and inducing fields. Journal of Experimental Psychology, 1953, 46, 453-456.

MacLeod, R. B. An experimental investigation of brightness constancy. Archives of Psychology, 1932, No. 135.

MacLeod, R. B. The effects of artificial penumbrae on the brightness of included areas. In Miscellanea Psychologia Albert Michotte, Louvain: Institut Superieur de Philosophie, 1947.

Mershon, D. H. Relative contributions of depth and directional adjacency to simultaneous whiteness contrast. Vision Research, 1972, 12, 969-979.

NoGUCHI, K., \& MasudA, N. Brightness changes in a complex field with changing illumination: A re-examination of Jameson and Hurvich's study of brightness constancy. Japanese Psychological Research, 1971, 13, 60-69.

Rock, I. In defense of unconscious inference. In W. Epstein (Ed.), Stability and constancy in visual perception: Mechanism and processes. New York: Wiley, 1977.

WALLACH, H. Brightness constancy and the nature of achromatic colors. Journal of Experimental Psychology, 1948, 38, 310-324.

Waldaven, J. Discounting the background: The missing link in the explanation of chromatic induction. Vision Research, $1976,16,289-296$.

Whittle, P., \& Challands, P. D. C. The effect of background luminance on the brightness of flashes. Vision Research, $1969,9,1095-1110$.

Yarbus, A. L. Eye movements and vision. New York: Plenum Press, 1967.

\section{NOTES}

1. It is widely written that Wallach's ratio effects apply only to a limited range of luminances. The main evidence for that view is contained in an experiment by Jameson and Hurvich (1961), who obtained lightness matches for each of a pattern of five gray squares under three levels of illumination. They reported that, as illumination increased, dark grays got blacker, light grays got whiter, and constancy held only for middle gray. This experiment has never been successfully replicated. Two failures to replicate have been published (Flock \& Noguchi, 1970; Noguchi \& Masuda, 1971). In addition, we have been consistently unable to reproduce the Jameson and Hurvich finding. We have repeated the experiment two times, under exactly the same conditions reported by Jameson and Hurvich, and both times we got almost perfect constancy for all five squares, with no decreasing functions.

2. Earlier reports had indicated that stabilized images either fade only partially or else fade and then reappear. Research with better methods of stabilization now makes it clear that the earlier findings were produced by a failure of complete stabilization (Barlow, 1963; Yarbus, 1967).

3. The slight perceptual changes known as contrast effects are tiny compared with the changes in edge ratios produced by changing the background color.

4. According to the edge integration concept, taken literally, the target disks ought to appear identical in reflectance, which they would not. But if edge information is primary, rather than luminance information, then the remarkable fact is not the slight perceived inequality of the two disks but, rather, how close the visual system comes to seeing the disks as equal by taking the background/background edge into account. In a subsequent paper, an explanation will be presented for why the integration of edges is never quite perfect, producing the contrast effect.

(Manuscript received April 12, 1982; revision accepted for publication January 20, 1983.) 